Article

\title{
Evaluation of the Life Cycle Greenhouse Gas Emissions from Hydroelectricity Generation Systems
}

\author{
Akhil Kadiyala ${ }^{1}$, Raghava Kommalapati ${ }^{1,2, *}$ and Ziaul Huque ${ }^{1,3}$ \\ 1 Center for Energy \& Environmental Sustainability, Prairie View A\&M University, Prairie View, TX 77446, \\ USA; akkadiyala@pvamu.edu (A.K.); zihuque@pvamu.edu (Z.H.) \\ 2 Department of Civil \& Environmental Engineering, Prairie View A\&M University, Prairie View, \\ TX 77446, USA \\ 3 Department of Mechanical Engineering, Prairie View A\&M University, Prairie View, TX 77446, USA \\ * Correspondence: rrkommalapati@pvamu.edu; Tel.: +1-936-261-1660
}

Academic Editor: Francesco Asdrubali

Received: 28 March 2016; Accepted: 1 June 2016; Published: 8 June 2016

\begin{abstract}
This study evaluated the life cycle greenhouse gas (GHG) emissions from different hydroelectricity generation systems by first performing a comprehensive review of the hydroelectricity generation system life cycle assessment (LCA) studies and then subsequent computation of statistical metrics to quantify the life cycle GHG emissions (expressed in grams of carbon dioxide equivalent per kilowatt hour, $\mathrm{gCO}_{2} \mathrm{e} / \mathrm{kWh}$ ). A categorization index (with unique category codes, formatted as "facility type-electric power generation capacity") was developed and used in this study to evaluate the life cycle GHG emissions from the reviewed hydroelectricity generation systems. The unique category codes were labeled by integrating the names of the two hydro power sub-classifications, i.e., the facility type (impoundment (I), diversion (D), pumped storage (PS), miscellaneous hydropower works (MHPW)) and the electric power generation capacity $($ micro $(\mu)$, small $(S)$, large $(L))$. The characterized hydroelectricity generation systems were statistically evaluated to determine the reduction in corresponding life cycle GHG emissions. A total of eight unique categorization codes (I-S, I-L, D- $\mu$, D-S, D-L, PS-L, MHPW- $\mu$, MHPW-S) were designated to the 19 hydroelectricity generation LCA studies (representing 178 hydropower cases) using the proposed categorization index. The mean life cycle GHG emissions resulting from the use of I-S $(\mathrm{N}=24), \mathrm{I}-\mathrm{L}(\mathrm{N}=8), \mathrm{D}-\mu(\mathrm{N}=3), \mathrm{D}-\mathrm{S}(\mathrm{N}=133), \mathrm{D}-\mathrm{L}(\mathrm{N}=3), \mathrm{PS}-\mathrm{L}(\mathrm{N}=3), \mathrm{MHPW}-\mu(\mathrm{N}=3)$, and MHPW-S ( $\mathrm{N}=1)$ hydroelectricity generation systems are $21.05 \mathrm{gCO}_{2} \mathrm{e} / \mathrm{kWh}, 40.63 \mathrm{gCO}_{2} \mathrm{e} / \mathrm{kWh}$, $47.82 \mathrm{gCO}_{2} \mathrm{e} / \mathrm{kWh}, 27.18 \mathrm{gCO}_{2} \mathrm{e} / \mathrm{kWh}, 3.45 \mathrm{gCO}_{2} \mathrm{e} / \mathrm{kWh}, 256.63 \mathrm{gCO}_{2} \mathrm{e} / \mathrm{kWh}, 19.73 \mathrm{gCO}_{2} \mathrm{e} / \mathrm{kWh}$, and $2.78 \mathrm{gCO}_{2} \mathrm{e} / \mathrm{kWh}$, respectively. D-L hydroelectricity generation systems produced the minimum life cycle GHGs (considering the hydroelectricity generation system categories with a representation of at least two cases).
\end{abstract}

Keywords: life cycle assessment; greenhouse gas emissions; hydro energy; impoundment; diversion; pumped storage; miscellaneous hydropower works; electricity generation

\section{Introduction}

Hydro energy may be defined as the energy harnessed from flowing water through a turbine to generate electricity. The principal components that help generate electricity from hydroelectric plants are the hydraulic turbine, the electric generator, the transformers and power house, and the reservoir [1]. A hydraulic turbine comprises of a row of blades connected to a rotating shaft. When the flowing water comes in contact with the blades of the turbine, the shaft of the hydraulic turbine is rotated due to its impact (change of velocity and pressure). The electric generator converts the mechanical energy from the rotation of the shaft into electric power by rotating a series of coils inside 
a magnetic field or vice versa, which results in the movement of electrons inside conductors that produces electric current. The transformers and power house of a hydropower plant act as an interface between the electric generator and the power transmission lines. The reservoirs store water before the movement through hydraulic turbines. The reservoir may be located either upstream (water flows under gravity) or downstream of the discharge end of the water turbine (water is pumped back).

The total electricity generation in 2012 across the world was reported to be 21.53 trillion kilowatt hours (kWh) [2]. The projected increase in world electricity generation for 2040 is 39 trillion $\mathrm{kWh}$ $(81 \%)$ [3]. The renewable energy sources have been projected to account for 9.6 trillion $\mathrm{kWh}(25 \%)$ of the world's total electricity generation in 2040. With the continuing depletion of traditional non-renewable energy sources, the necessity for generating electricity through the use of renewable energy sources (hydro, wind, biomass, solar, geothermal) increased significantly. Based on the 2012 statistics, hydro energy was identified to be the largest renewable energy source for electricity generation with a contribution of 3.646 trillion $\mathrm{kWh}$ that accounted for $16.94 \%$ of the world's total electricity generated in 2012 [2].

Considering the significant role that hydroelectricity generation systems have to play in meeting the world's electrical energy demand, one needs to evaluate the life cycle greenhouse gas (GHG) emissions resulting from the adoption of different categories of hydroelectricity generation systems. The life cycle assessment (LCA) approach helps evaluate the net carbon dioxide $\left(\mathrm{CO}_{2}\right)$ emissions resulting from the use of hydro energy as a fuel. LCA is an analytical method that provides an assessment of the environmental impacts of the considered products and technologies from a "cradle to grave" systems perspective utilizing the detailed input and output parameters that operate within the designated system boundaries.

Many studies [4-22] analyzed the LCA of hydroelectricity generation systems. The use of hydroelectricity generation systems across the world is being encouraged considering that water is a free and an abundant renewable energy resource. The development of hydroelectricity generation systems provides additional benefits such as the development of recreational opportunities (fishing, swimming, boating), flood control, irrigation, water supply, and back-up power during major electricity outages or disruptions. The use of hydroelectricity generation systems has the disadvantages of impacting the fish population (with restrictions on the movement of fish between upstream and downstream water bodies), low dissolved oxygen levels in the water that impacts the riverbank habitats, drought conditions limiting the water supply, and high construction costs leading to higher payback time for economical returns. A more detailed description of the LCA boundary conditions, GHG emissions, and site-specific characteristics associated with each of the aforementioned hydroelectricity generation system studies are provided in the section titled "Review of Hydro LCA Studies".

All the hydro LCA publications to date have emphasized on the determination of the life cycle GHG emissions for site-specific hydroelectricity generation system installations. There are limited studies [23-26] that summarized the life cycle GHG emissions from hydroelectricity generation systems. None of the earlier LCA review studies evaluated and compared the life cycle GHG emissions across a distinct set of hydroelectricity generation system categories. This study aims to fill this knowledge gap by performing a comprehensive review of the literature on all the currently available hydroelectricity generation LCA studies, followed by a statistical evaluation of the life cycle GHG emissions from the reviewed hydroelectricity generation system categories distinctly. The results from the statistical evaluation of the life cycle GHG emissions will assist energy policy makers and environmental professionals in identifying and encouraging the use of environmental-friendly hydroelectricity generation system category options to generate electricity with minimal GHG emissions. 


\section{Methodology}

A review of the literature showed that the hydroelectricity generation systems may be categorized on the basis of the type of facility and the electric power generation capacity. The classification of hydroelectricity generation systems on the basis of facility type [27] is as follows:

- impoundment (I)-The impoundment is the most common type of facility. The facility has a dam that stores water in the reservoir and generates electricity when moving water rotates the turbines.

- diversion (D) - The diversion facility, also referred to as the run-of-river facility, diverts a section of river water through a canal or penstock to generate electricity. The use of a dam is not mandatory in the diversion facility.

- $\quad$ pumped storage (PS) - The pumped storage facility uses energy from other sources (e.g., solar, wind, etc.) to pump water uphill from a lower reservoir to an upper reservoir during periods of low energy demand. Water from the upper reservoir flows back into the lower reservoir during periods of high energy demand and generates electricity.

In addition to the aforementioned facility-based classification systems, this study considered the category of miscellaneous hydropower works (MHPW), which utilizes existing water infrastructures such as the water distribution networks, canal locks, compensation flows, water treatment works, etc., to generate electricity in small quantities that can either be used on-site or transmitted for consumption by a small community locally.

Depending on the electric power generation capacity, hydroelectricity generation systems may be categorized [27] as follows:

- $\operatorname{micro}(\mu)$-electricity generation capacity is less than 0.1 megawatt (MW).

- $\quad$ small (S) —electricity generation capacity is between than 0.1 and $30 \mathrm{MW}$.

- $\quad$ large (L)-electricity generation capacity is greater than $30 \mathrm{MW}$.

This study proposed and adopted the use of a new categorization index that integrated the names of both the classification systems mentioned above to generate unique category codes, represented by "facility type-electric power generation capacity" labels to categorize the hydroelectricity generation systems. A total of 12 unique category codes (I- $\mu, \mathrm{I}-\mathrm{S}, \mathrm{I}-\mathrm{L}, \mathrm{D}-\mu, \mathrm{D}-\mathrm{S}, \mathrm{D}-\mathrm{L}, \mathrm{PS}-\mu, \mathrm{PS}-\mathrm{S}, \mathrm{PS}-\mathrm{L}, \mathrm{MHPW}-\mu$, MHPW-S, MHPW-L) may be generated using different combinations of the two classification systems. Each of the reviewed hydro LCA studies was first assigned one of the unique category codes proposed in this study. Next, the life cycle GHG emissions from the individual category coded hydroelectricity generation systems were evaluated using statistical metrics (sample size, mean, standard deviation, minimum, maximum, standard error of the mean, quartile 1, quartile 2 or median, quartile 3) and graphical representations (error bars representing the mean with $95 \%$ confidence intervals, box plots representing the quartiles with outliers). While the error bars demonstrate the degree of confidence in the mean GHG emissions, the box plots provide information on the degree of variation among the LCA studies characterized by different categories of hydroelectricity generation systems.

\section{Results and Discussion}

\subsection{Review of Hydro LCA Studies}

There are several studies [4-22] that evaluated the life cycle environmental impacts of using hydro energy for electricity generation. One has to define the system boundary conditions (that includes details on the activities or processes to be considered in the analysis) and a functional unit of measure (that enables quantification of the net environmental impacts from carrying out an activity or a process as defined within the LCA system boundary conditions) when performing a LCA.

The majority of the reviewed LCA studies $[9,14,15,18-20,22]$ considered the system boundaries that included activities related to the construction, operation, and maintenance of the hydroelectricity 
generation systems. Other studies $[7,11,13,16,17]$ adopted the system boundaries to include the raw material extraction, material processing, component manufacturing process, transportation, assembly and installation, operation and maintenance, and end-of-life process (decommissioning, recycling/reuse, and final disposal) activities. Gallagher et al. [21] excluded the end-of-life process activity and considered the remaining activities listed within the system boundaries defined by other studies $[7,11,13,16,17]$. Ribeiro and Silva [10] considered the construction site operations, emissions from reservoir flooding, and worker transportation activities within their system boundaries in addition to those associated with other studies [7,11,13,16,17]. Gagnon and van de Vate [4] performed the LCA of hydroelectricity generation systems with system boundaries that considered all the activities related to construction, the preparation of flooded land (biomass decay in the flooded lands), and the use of thermal backup power. Denholm and Kulcinski [5], and Suwanit and Gheewala [12] had system boundary conditions that included the activities of land preparation before construction, construction, operation and maintenance, and decommissioning. Hondo [6] considered only electric generation activity as the system boundary when performing the LCA of a hydroelectricity generation system. Rule et al. [8] considered the activities of construction, transportation, and decommissioning $(50 \%$ recycling $+50 \%$ landfill for materials that are recyclable; $100 \%$ landfill for all other materials) as the system boundaries in their LCA.

The common functional unit of measure adopted by the majority of the hydro LCA studies is grams of $\mathrm{CO}_{2}$ equivalent per kilowatt hour $\left(\mathrm{gCO}_{2} \mathrm{e} / \mathrm{kWh}\right)$ of electricity produced. Accordingly, this study also adopts the functional unit of measure for GHG emissions to be $\mathrm{gCO}_{2} \mathrm{e} / \mathrm{kWh}$ of electricity produced.

Table 1 provides a summary of the hydroelectricity generation system categorization (unique category codes generated from the combination of the two classification systems-facility type (I, D, PS, MHPW), electric power generation capacity $(\mu, \mathrm{S}, \mathrm{L})$ along with the corresponding GHG emissions (in $\mathrm{gCO}_{2} \mathrm{e} / \mathrm{kWh}$ ) for the reviewed hydro studies. Table 1 also provides additional site-specific details that included the electricity generation capacity (EGC, expressed in MW), the capacity factor (CF, expressed in \%), the hydropower plant life (PL, in years), and the geographical location (GL) for the installed hydroelectricity generation systems. EGC is the amount of electrical power that is produced under standard operating conditions. CF is the ratio of the average power generated to the rated peak power.

Based on the review of 19 hydroelectricity generation LCA studies (refer to Table 1), one may note that the D-S $(\mathrm{N}=133)$ hydroelectricity generation systems were more in number compared to I-S $(\mathrm{N}=24), \mathrm{I}-\mathrm{L}(\mathrm{N}=8), \mathrm{D}-\mu(\mathrm{N}=3), \mathrm{D}-\mathrm{L}(\mathrm{N}=3)$, PS-L $(\mathrm{N}=3), \mathrm{MHPW}-\mu(\mathrm{N}=3)$, and MHPW-S $(\mathrm{N}=1)$ hydroelectricity generation systems. There were no LCA studies on the use of I- $\mu$, PS- $\mu$, PS-S, and MHPW-L hydroelectricity generation systems. This is because the capital investments associated with the construction and maintenance of the impoundment and pumped storage hydroelectricity generation systems are considerably higher, which makes the generation of electricity at micro and small scale levels to not be feasible. The miscellaneous hydropower works are not feasible for large-scale electricity generation as they are designed for optimization and generation of electricity utilizing existing waterworks in small quantities for localized consumption. From Table 1, one may also note that the diversion hydroelectricity generation systems $(\mathrm{N}=139)$ were more in number compared to the impoundment $(\mathrm{N}=32)$, the miscellaneous hydropower works $(\mathrm{N}=4)$, and the pumped storage $(\mathrm{N}=3)$ systems, and small hydroelectricity generation systems $(\mathrm{N}=158)$ were more in number compared to the large hydroelectricity generation systems $(\mathrm{N}=14)$ and the micro hydroelectricity generation systems $(\mathrm{N}=6)$. 
Table 1. GHG emissions from hydroelectricity generation systems.

\begin{tabular}{|c|c|c|c|c|c|}
\hline \multirow[b]{2}{*}{ Source } & \multicolumn{3}{|c|}{ Categorization } & \multirow{2}{*}{$\begin{array}{l}\text { GHG Emissions } \\
\left(\mathrm{gCO}_{2} \mathrm{e} / \mathrm{kWh}\right)\end{array}$} & \multirow{2}{*}{$\begin{array}{c}\text { Additional Features } \\
\text { EGC (MW); CF (\%); PL (years); GL }\end{array}$} \\
\hline & $\begin{array}{l}\text { Facility } \\
\text { Type }\end{array}$ & $\begin{array}{c}\text { Electric Power } \\
\text { Generation Capacity }\end{array}$ & $\begin{array}{c}\text { Unique } \\
\text { Category Code }\end{array}$ & & \\
\hline Gagnon and van de Vate [4] & I & $\mathrm{L}$ & $\mathrm{I}-\mathrm{L}$ & 237 & $\mathrm{EGC}=4000 ; \mathrm{PL}=100 ; \mathrm{GL}=$ Japan \\
\hline Denholm and Kulcinski [5] & PS & $\mathrm{L}$ & PS-L & 5.6 & $\mathrm{EGC}=31-2100 ; \mathrm{CF}=20 ; \mathrm{PL}=60 ; \mathrm{GL}=\mathrm{USA}$ \\
\hline Hondo [6] & $\mathrm{D}$ & $\mathrm{S}$ & D-S & 11.3 & $\mathrm{EGC}=10 ; \mathrm{CF}=45 ; \mathrm{PL}=30 ; \mathrm{GL}=$ Japan \\
\hline Pehnt [7] & $\begin{array}{l}\mathrm{D} \\
\mathrm{D}\end{array}$ & $\begin{array}{l}S \\
S\end{array}$ & $\begin{array}{l}\text { D-S } \\
\text { D-S }\end{array}$ & $\begin{array}{l}10 \\
13\end{array}$ & $\begin{aligned} \text { EGC } & =3.1 ; \mathrm{GL}=\text { Germany } \\
\mathrm{EGC} & =0.3 ; \mathrm{GL}=\text { Germany }\end{aligned}$ \\
\hline Rule et al. [8] & I & $\mathrm{L}$ & $\mathrm{I}-\mathrm{L}$ & 4.6 & EGC $=432(4 \times 108) ; \mathrm{PL}=100 ; \mathrm{GL}=$ New Zealand \\
\hline Environmental Product Declaration Report [9] & $\mathrm{D}$ & $\mathrm{L}$ & D-L & 3.16 & $\mathrm{EGC}=50 ; \mathrm{PL}=100 ; \mathrm{GL}=$ Aargau, Switzerland \\
\hline Ribeiro and Silva [10] & I & $\mathrm{L}$ & $\mathrm{I}-\mathrm{L}$ & 1.56 & $\mathrm{EGC}=14000 ; \mathrm{PL}=100 ; \mathrm{GL}=$ Brazil-Paraguay \\
\hline Pascale et al. [11] & MHPW & $\mu$ & MHPW- $\mu$ & 52.7 & EGC $=0.003 ; C F=53 ; P L=20 ; G L=$ Thailand \\
\hline Suwanit and Gheewala [12] & $\begin{array}{l}\mathrm{D} \\
\mathrm{D} \\
\mathrm{D} \\
\mathrm{D} \\
\mathrm{D}\end{array}$ & $\begin{array}{l}\mathrm{S} \\
\mathrm{S} \\
\mathrm{S} \\
\mathrm{S} \\
\mathrm{S}\end{array}$ & $\begin{array}{l}\text { D-S } \\
\text { D-S } \\
\text { D-S } \\
\text { D-S } \\
\text { D-S }\end{array}$ & $\begin{array}{l}11.01 \\
23.01 \\
16.28 \\
22.71 \\
16.49\end{array}$ & $\begin{array}{l}\mathrm{EGC}=5.1 ; \mathrm{PL}=100 ; \mathrm{GL}=\text { Thailand } \\
\mathrm{EGC}=6(2 \times 3) ; \mathrm{PL}=100 ; \mathrm{GL}=\text { Thailand } \\
\mathrm{EGC}=2.5(2 \times 1.25) ; \mathrm{PL}=100 ; \mathrm{GL}=\text { Thailand } \\
\mathrm{EGC}=2.25 ; \mathrm{PL}=100 ; \mathrm{GL}=\text { Thailand } \\
\mathrm{EGC}=1.15 ; \mathrm{PL}=100 ; \mathrm{GL}=\text { Thailand }\end{array}$ \\
\hline Flury and Frischknecht [13] & $\begin{array}{l}\text { I } \\
\text { I } \\
\text { PS } \\
\text { PS } \\
\text { D } \\
\text { D }\end{array}$ & $\begin{array}{l}\text { L } \\
\text { L } \\
\text { L } \\
\text { L } \\
\text { S } \\
\text { S }\end{array}$ & $\begin{array}{l}\text { I-L } \\
\text { I-L } \\
\text { PS-L } \\
\text { PS-L } \\
\text { D-S } \\
\text { D-S }\end{array}$ & $\begin{array}{c}5.86 \\
16.64 \\
155.1 \\
609.2 \\
3.62 \\
3.79\end{array}$ & $\begin{array}{l}\text { EGC }=95 ; \mathrm{PL}=150 ; \mathrm{GL}=\text { alpine, Europe } \\
\mathrm{EGC}=95 ; \mathrm{PL}=150 ; \mathrm{GL}=\text { non-alpine, Europe } \\
\mathrm{EGC}=95 ; \mathrm{PL}=150 ; \mathrm{GL}=\text { Switzerland } \\
\mathrm{EGC}=95 ; \mathrm{PL}=150 ; \mathrm{GL}=\text { Europe } \\
\mathrm{EGC}=8.6 ; \mathrm{PL}=80 ; \mathrm{GL}=\text { Switzerland } \\
\mathrm{EGC}=8.6 ; \mathrm{PL}=80 ; \mathrm{GL}=\text { Europe }\end{array}$ \\
\hline National Energy Technology Laboratory Report [14] & I & $\mathrm{L}$ & $\mathrm{I}-\mathrm{L}$ & 43.8 & $\mathrm{EGC}=2080 ; \mathrm{PL}=80 ; \mathrm{GL}=\mathrm{USA}$ \\
\hline Varun et al. [15] & $\begin{array}{l}\mathrm{D} \\
\mathrm{D} \\
\mathrm{D} \\
\mathrm{D} \\
\mathrm{D} \\
\mathrm{D} \\
\mathrm{D} \\
\mathrm{D} \\
\mathrm{D} \\
\mathrm{D} \\
\mathrm{D} \\
\mathrm{D}\end{array}$ & $\begin{array}{l}\text { S } \\
\text { S } \\
\text { S } \\
\text { S } \\
\text { S } \\
\text { S } \\
\text { S } \\
\text { S } \\
\text { S } \\
\text { S } \\
\text { S } \\
\text { S }\end{array}$ & $\begin{array}{l}\text { D-S } \\
\text { D-S } \\
\text { D-S } \\
\text { D-S } \\
\text { D-S } \\
\text { D-S } \\
\text { D-S } \\
\text { D-S } \\
\text { D-S } \\
\text { D-S } \\
\text { D-S } \\
\text { D-S }\end{array}$ & $\begin{array}{l}26.41 \\
25.52 \\
28.14 \\
30.33 \\
16.44 \\
15.44 \\
15.85 \\
31.19 \\
24.19 \\
19.99 \\
31.11 \\
29.97\end{array}$ & $\begin{array}{l}\mathrm{EGC}=1 ; \mathrm{PL}=30 ; \mathrm{GL}=\text { Karnataka, India } \\
\mathrm{EGC}=1.5 ; \mathrm{PL}=30 ; \mathrm{GL}=\text { Karnataka, India } \\
\mathrm{EGC}=1 ; \mathrm{PL}=30 ; \mathrm{GL}=\text { Karnataka, India } \\
\mathrm{EGC}=0.25 ; \mathrm{PL}=30 ; \mathrm{GL}=\text { Karnataka, India } \\
\mathrm{EGC}=4.5 ; \mathrm{PL}=30 ; \mathrm{GL}=\text { Rajasthan, India } \\
\mathrm{EGC}=4.5 ; \mathrm{PL}=30 ; \mathrm{GL}=\text { Rajasthan, India } \\
\mathrm{EGC}=4(2 \times 2) ; \mathrm{PL}=30 ; \mathrm{GL}=\text { Rajasthan, India } \\
\mathrm{EGC}=0.85 ; \mathrm{PL}=30 ; \mathrm{GL}=\text { Karnataka, India } \\
\mathrm{EGC}=1.5 ; \mathrm{PL}=30 ; \mathrm{GL}=\text { Andhra Pradesh, India } \\
\mathrm{EGC}=3 ; \mathrm{PL}=30 ; \mathrm{GL}=\text { Karnataka, India } \\
\mathrm{EGC}=0.8(2 \times 0.4) ; \mathrm{PL}=30 ; \mathrm{GL}=\text { Andhra Pradesh, India } \\
\mathrm{EGC}=0.8 ; \mathrm{PL}=30 ; \mathrm{GL}=\text { Andhra Pradesh, India }\end{array}$ \\
\hline
\end{tabular}


Table 1. Cont.

\begin{tabular}{|c|c|c|c|c|c|}
\hline \multirow[b]{2}{*}{ Source } & \multicolumn{3}{|c|}{ Categorization } & \multirow{2}{*}{$\begin{array}{l}\text { GHG Emissions } \\
\left(\mathrm{gCO}_{2} \mathrm{e} / \mathrm{kWh}\right)\end{array}$} & \multirow{2}{*}{$\begin{array}{c}\text { Additional Features } \\
\text { EGC (MW); CF (\%); PL (years); GL }\end{array}$} \\
\hline & $\begin{array}{l}\text { Facility } \\
\text { Type }\end{array}$ & $\begin{array}{c}\text { Electric Power } \\
\text { Generation Capacity }\end{array}$ & $\begin{array}{c}\text { Unique } \\
\text { Category Code }\end{array}$ & & \\
\hline \multirow{37}{*}{ Varun et al. [15] } & $\mathrm{D}$ & $\mathrm{S}$ & D-S & 27.96 & $\mathrm{EGC}=2(2 \times 1) ; \mathrm{PL}=30 ; \mathrm{GL}=$ Andhra Pradesh, India \\
\hline & $\mathrm{D}$ & $\mathrm{S}$ & D-S & 25.27 & $\mathrm{EGC}=2(2 \times 1) ; \mathrm{PL}=30 ; \mathrm{GL}=$ Andhra Pradesh, India \\
\hline & $\mathrm{D}$ & $\mathrm{S}$ & D-S & 33.12 & $E G C=0.65 ; P L=30 ; G L=$ Andhra Pradesh, India \\
\hline & $\mathrm{D}$ & $\mathrm{S}$ & D-S & 28.75 & $\mathrm{EGC}=0.8 ; \mathrm{PL}=30 ; \mathrm{GL}=$ Andhra Pradesh, India \\
\hline & $\mathrm{D}$ & $\mathrm{S}$ & D-S & 23.8 & $E G C=2(2 \times 1) ; P L=30 ; G L=$ Andhra Pradesh, India \\
\hline & $\mathrm{D}$ & $\mathrm{S}$ & D-S & 26.33 & $\mathrm{EGC}=2.14(2 \times 1.07) ; \mathrm{PL}=30 ; \mathrm{GL}=$ Andhra Pradesh, India \\
\hline & $\mathrm{D}$ & $\mathrm{S}$ & D-S & 28.67 & $\mathrm{EGC}=1.588(2 \times 0.794) ; \mathrm{PL}=30 ; \mathrm{GL}=$ Andhra Pradesh, India \\
\hline & $\mathrm{D}$ & S & D-S & 26.16 & $\mathrm{EGC}=2.47(2 \times 1.235) ; \mathrm{PL}=30 ; \mathrm{GL}=$ Andhra Pradesh, India \\
\hline & $\mathrm{D}$ & $\mathrm{S}$ & D-S & 35.97 & $\mathrm{EGC}=0.5 ; \mathrm{PL}=30 ; \mathrm{GL}=$ Andhra Pradesh, India \\
\hline & $\mathrm{D}$ & $\mathrm{S}$ & D-S & 37.56 & $\mathrm{EGC}=0.5 ; \mathrm{PL}=30 ; \mathrm{GL}=$ Andhra Pradesh, India \\
\hline & $\mathrm{D}$ & $\mathrm{S}$ & D-S & 38.25 & $\mathrm{EGC}=0.55 ; \mathrm{PL}=30 ; \mathrm{GL}=$ Andhra Pradesh, India \\
\hline & $\mathrm{D}$ & $\mathrm{S}$ & D-S & 35.64 & $\mathrm{EGC}=0.55 ; \mathrm{PL}=30 ; \mathrm{GL}=$ Andhra Pradesh, India \\
\hline & $\mathrm{D}$ & $\mathrm{S}$ & D-S & 33.44 & $\mathrm{EGC}=0.5 ; \mathrm{PL}=30 ; \mathrm{GL}=$ Andhra Pradesh, India \\
\hline & $\mathrm{D}$ & $\mathrm{S}$ & D-S & 27.1 & $\mathrm{EGC}=3.3(2 \times 1.65) ; \mathrm{PL}=30 ; \mathrm{GL}=$ Andhra Pradesh, India \\
\hline & $\mathrm{D}$ & $\mathrm{S}$ & D-S & 32.71 & $\mathrm{EGC}=0.5 ; \mathrm{PL}=30 ; \mathrm{GL}=$ Andhra Pradesh, India \\
\hline & $\mathrm{D}$ & $\mathrm{S}$ & D-S & 30.43 & EGC $=0.8(2 \times 0.4) ; \mathrm{PL}=30 ; \mathrm{GL}=$ Andhra Pradesh, India \\
\hline & $\mathrm{D}$ & $\mathrm{S}$ & D-S & 23.34 & $\mathrm{EGC}=2(2 \times 1) ; \mathrm{PL}=30 ; \mathrm{GL}=$ Andhra Pradesh, India \\
\hline & $\mathrm{D}$ & $\mathrm{S}$ & D-S & 32.37 & $\mathrm{EGC}=0.55 ; \mathrm{PL}=30 ; \mathrm{GL}=$ Tamilnadu, India \\
\hline & $\mathrm{D}$ & $\mathrm{S}$ & D-S & 29.56 & $\mathrm{EGC}=0.8 ; \mathrm{PL}=30 ; \mathrm{GL}=$ Andhra Pradesh, India \\
\hline & $\mathrm{D}$ & $\mathrm{S}$ & D-S & 26.51 & $\mathrm{EGC}=3.3(2 \times 1.65) ; \mathrm{PL}=30 ; \mathrm{GL}=$ Andhra Pradesh, India \\
\hline & $\mathrm{D}$ & $\mathrm{S}$ & D-S & 20.56 & $\mathrm{EGC}=7.5(3 \times 2.5) ; \mathrm{PL}=30 ; \mathrm{GL}=$ Andhra Pradesh, India \\
\hline & $\mathrm{D}$ & $\mathrm{S}$ & D-S & 28.92 & $\mathrm{EGC}=1 ; \mathrm{PL}=30 ; \mathrm{GL}=$ Andhra Pradesh, India \\
\hline & $\mathrm{D}$ & $\mathrm{S}$ & D-S & 32.71 & $E G C=0.65 ; P L=30 ; G L=$ Andhra Pradesh, India \\
\hline & $\mathrm{D}$ & $\mathrm{S}$ & D-S & 23.74 & $\mathrm{EGC}=1.85 ; \mathrm{PL}=30 ; \mathrm{GL}=$ Andhra Pradesh, India \\
\hline & $\mathrm{D}$ & $\mathrm{S}$ & D-S & 24.89 & $\mathrm{EGC}=1.7 ; \mathrm{PL}=30 ; \mathrm{GL}=$ Andhra Pradesh, India \\
\hline & $\mathrm{D}$ & $\mathrm{S}$ & D-S & 25.42 & $\mathrm{EGC}=1.7 ; \mathrm{PL}=30 ; \mathrm{GL}=$ Andhra Pradesh, India \\
\hline & $\mathrm{D}$ & $\mathrm{S}$ & D-S & 20.39 & $\mathrm{EGC}=3.75(3 \times 1.25) ; \mathrm{PL}=30 ; \mathrm{GL}=$ Andhra Pradesh, India \\
\hline & $\mathrm{D}$ & $\mathrm{s}$ & D-S & 28.17 & $\mathrm{EGC}=1(2 \times 0.5) ; \mathrm{PL}=30 ; \mathrm{GL}=$ Bihar, India \\
\hline & $\mathrm{D}$ & $\mathrm{S}$ & D-S & 33.31 & $\mathrm{EGC}=0.7(2 \times 0.35) ; \mathrm{PL}=30 ; \mathrm{GL}=$ Bihar, India \\
\hline & $\mathrm{D}$ & $\mathrm{S}$ & D-S & 28.18 & $\mathrm{EGC}=1(2 \times 0.5) ; \mathrm{PL}=30 ; \mathrm{GL}=$ Bihar, India \\
\hline & $\mathrm{D}$ & $\mathrm{S}$ & D-S & 31.56 & $\mathrm{EGC}=0.5 ; \mathrm{PL}=30 ; \mathrm{GL}=$ Bihar, India \\
\hline & $\mathrm{D}$ & $\mathrm{S}$ & D-S & 41.75 & $\mathrm{EGC}=0.25 ; \mathrm{PL}=30 ; \mathrm{GL}=$ Bihar, India \\
\hline & $\mathrm{D}$ & $\mathrm{S}$ & D-S & 28.19 & $\mathrm{EGC}=1(2 \times 0.5) ; \mathrm{PL}=30 ; \mathrm{GL}=$ Bihar, India \\
\hline & $\mathrm{D}$ & $\mathrm{S}$ & D-S & 39.77 & $\mathrm{EGC}=0.5(2 \times 0.25) ; \mathrm{PL}=30 ; \mathrm{GL}=$ Bihar, India \\
\hline & $\mathrm{D}$ & $\mathrm{S}$ & D-S & 23.38 & $\mathrm{EGC}=1.5(2 \times 0.75) ; \mathrm{PL}=30 ; \mathrm{GL}=$ Bihar, India \\
\hline & $\mathrm{D}$ & $\mathrm{S}$ & D-S & 26.12 & $\mathrm{EGC}=1(2 \times 0.5) ; \mathrm{PL}=30 ; \mathrm{GL}=$ Bihar, India \\
\hline & $\mathrm{D}$ & $\mathrm{s}$ & D-S & 21.2 & $\mathrm{EGC}=3(2 \times 1.5) ; \mathrm{PL}=30 ; \mathrm{GL}=$ Karnataka, India \\
\hline
\end{tabular}


Table 1. Cont.

\begin{tabular}{|c|c|c|c|c|c|}
\hline \multirow[b]{2}{*}{ Source } & \multicolumn{3}{|c|}{ Categorization } & \multirow{2}{*}{$\begin{array}{l}\text { GHG Emissions } \\
\left(\mathrm{gCO}_{2} \mathrm{e} / \mathrm{kWh}\right)\end{array}$} & \multirow{2}{*}{$\begin{array}{c}\text { Additional Features } \\
\text { EGC (MW); CF (\%); PL (years); GL }\end{array}$} \\
\hline & $\begin{array}{l}\text { Facility } \\
\text { Type }\end{array}$ & $\begin{array}{c}\text { Electric Power } \\
\text { Generation Capacity }\end{array}$ & $\begin{array}{c}\text { Unique } \\
\text { Category Code }\end{array}$ & & \\
\hline \multirow{36}{*}{ Varun et al. [15] } & $\mathrm{D}$ & $\mathrm{S}$ & D-S & 22.15 & $\mathrm{EGC}=2.8 ; \mathrm{PL}=30 ; \mathrm{GL}=$ Karnataka, India \\
\hline & $\mathrm{D}$ & $\mathrm{s}$ & D-S & 24.29 & $\mathrm{EGC}=1.3 ; \mathrm{PL}=30 ; \mathrm{GL}=$ Karnataka, India \\
\hline & $\mathrm{D}$ & $\mathrm{S}$ & D-S & 36.4 & $\mathrm{EGC}=0.25 ; \mathrm{PL}=30 ; \mathrm{GL}=$ Madhya Pradesh, India \\
\hline & $\mathrm{D}$ & $\mathrm{s}$ & D-S & 28.07 & $\mathrm{EGC}=1(2 \times 0.5) ; \mathrm{PL}=30 ; \mathrm{GL}=$ Madhya Pradesh, India \\
\hline & $\mathrm{D}$ & $\mathrm{S}$ & D-S & 32.02 & EGC $=1.4(2 \times 0.7) ; \mathrm{PL}=30 ; \mathrm{GL}=$ Punjab, India \\
\hline & $\mathrm{D}$ & $\mathrm{S}$ & D-S & 32.05 & $\mathrm{EGC}=1.5(2 \times 0.75) ; \mathrm{PL}=30 ; \mathrm{GL}=$ Punjab, India \\
\hline & $\mathrm{D}$ & $\mathrm{s}$ & D-S & 32.05 & $\mathrm{EGC}=1.5(2 \times 0.75) ; \mathrm{PL}=30 ; \mathrm{GL}=$ Punjab, India \\
\hline & $\mathrm{D}$ & $\mathrm{S}$ & D-S & 31.04 & EGC $=1.5(2 \times 0.75) ; \mathrm{PL}=30 ; \mathrm{GL}=$ Punjab, India \\
\hline & $\mathrm{D}$ & $\mathrm{s}$ & D-S & 35.12 & $\mathrm{EGC}=0.535 ; \mathrm{PL}=30 ; \mathrm{GL}=$ Rajasthan, India \\
\hline & $\mathrm{D}$ & $\mathrm{s}$ & D-S & 27.55 & $\mathrm{EGC}=1.2 ; \mathrm{PL}=30 ; \mathrm{GL}=$ Rajasthan, India \\
\hline & $\mathrm{D}$ & $\mathrm{S}$ & D-S & 22.85 & EGC $=2.25(3 \times 0.75) ; \mathrm{PL}=30 ; \mathrm{GL}=$ Tamilnadu, India \\
\hline & $\mathrm{D}$ & $\mathrm{s}$ & D-S & 31.29 & $\mathrm{EGC}=0.75 ; \mathrm{PL}=30 ; \mathrm{GL}=$ West Bengal, India \\
\hline & I & $\mathrm{s}$ & $\mathrm{I}-\mathrm{S}$ & 31.2 & $\mathrm{EGC}=1(2 \times 0.5) ; \mathrm{PL}=30 ; \mathrm{GL}=$ Bihar, India \\
\hline & I & $\mathrm{S}$ & I-S & 33.86 & $\mathrm{EGC}=0.4(2 \times 0.2) ; \mathrm{PL}=30 ; \mathrm{GL}=$ Bihar, India \\
\hline & I & $\mathrm{s}$ & I-S & 23.9 & $\mathrm{EGC}=15 ; \mathrm{PL}=30 ; \mathrm{GL}=$ Maharashtra, India \\
\hline & I & $\mathrm{s}$ & I-S & 25.6 & EGC $=1.5(2 \times 0.75) ; \mathrm{PL}=30 ; \mathrm{GL}=$ Karnataka, India \\
\hline & I & $\mathrm{S}$ & I-S & 16.63 & EGC = 10; PL = 30; GL = Andhra Pradesh, India \\
\hline & I & $\mathrm{s}$ & I-S & 26.6 & $\mathrm{EGC}=1.5 ; \mathrm{PL}=30 ; \mathrm{GL}=$ Karnataka, India \\
\hline & I & $\mathrm{s}$ & I-S & 21.46 & $\mathrm{EGC}=2 ; \mathrm{PL}=30 ; \mathrm{GL}=$ Andhra Pradesh, India \\
\hline & I & $\mathrm{s}$ & I-S & 15.1 & $\mathrm{EGC}=15(2 \times 7.5) ; \mathrm{PL}=30 ; \mathrm{GL}=$ Andhra Pradesh, India \\
\hline & I & $\mathrm{s}$ & I-S & 17.6 & $\mathrm{EGC}=2.4 ; \mathrm{PL}=30 ; \mathrm{GL}=$ Karnataka, India \\
\hline & I & $\mathrm{s}$ & I-S & 19.39 & EGC $=2(2 \times 1) ; \mathrm{PL}=30 ; \mathrm{GL}=$ Karnataka, India \\
\hline & I & $\mathrm{S}$ & I-S & 16.4 & $\mathrm{EGC}=9(2 \times 4.5) ; \mathrm{PL}=30 ; \mathrm{GL}=$ Karnataka, India \\
\hline & I & $\mathrm{s}$ & I-S & 15.25 & $\mathrm{EGC}=16(4 \times 4) ; \mathrm{PL}=30 ; \mathrm{GL}=$ Karnataka, India \\
\hline & I & $\mathrm{s}$ & I-S & 21.57 & $\mathrm{EGC}=2.4(2 \times 1.2) ; \mathrm{PL}=30 ; \mathrm{GL}=$ Madhya Pradesh, India \\
\hline & I & $\mathrm{s}$ & I-S & 12.76 & EGC $=16(2 \times 8) ; \mathrm{PL}=30 ; \mathrm{GL}=$ Maharashtra, India \\
\hline & I & $\mathrm{s}$ & I-S & 11.34 & $\mathrm{EGC}=16 ; \mathrm{PL}=30 ; \mathrm{GL}=$ Maharashtra, India \\
\hline & I & $\mathrm{s}$ & I-S & 27.5 & EGC $=1.3(2 \times 0.65) ; \mathrm{PL}=30 ; \mathrm{GL}=$ Tamilnadu, India \\
\hline & I & $\mathrm{s}$ & I-S & 18.62 & EGC $=2.5(2 \times 0.125) ; \mathrm{PL}=30 ; \mathrm{GL}=$ Tamilnadu, India \\
\hline & I & $\mathrm{s}$ & I-S & 30.99 & EGC $=0.7(2 \times 0.35) ; \mathrm{PL}=30 ; \mathrm{GL}=$ Tamilnadu, India \\
\hline & I & $\mathrm{S}$ & I-S & 23.76 & $\mathrm{EGC}=2 ; \mathrm{PL}=30 ; \mathrm{GL}=$ Tamilnadu, India \\
\hline & I & $\mathrm{s}$ & I-S & 13.53 & $\mathrm{EGC}=9(2 \times 4.5) ; \mathrm{PL}=30 ; \mathrm{GL}=$ Maharashtra, India \\
\hline & I & $\mathrm{s}$ & I-S & 26.06 & $\mathrm{EGC}=1.5 ; \mathrm{PL}=30 ; \mathrm{GL}=$ Maharashtra, India \\
\hline & I & $\mathrm{S}$ & I-S & 20.58 & $\mathrm{EGC}=1.5 ; \mathrm{PL}=30 ; \mathrm{GL}=$ Karnataka, India \\
\hline & I & $\mathrm{s}$ & I-S & 21.44 & $E G C=3(2 \times 1.5) ; \mathrm{PL}=30 ; \mathrm{GL}=$ Andhra Pradesh, India \\
\hline & I & $\mathrm{s}$ & I-S & 14.13 & $\mathrm{EGC}=8(2 \times 4) ; \mathrm{PL}=30 ; \mathrm{GL}=$ Karnataka, India \\
\hline
\end{tabular}


Table 1. Cont.

\begin{tabular}{|c|c|c|c|c|c|}
\hline \multirow[b]{2}{*}{ Source } & \multicolumn{3}{|c|}{ Categorization } & \multirow{2}{*}{$\begin{array}{l}\text { GHG Emissions } \\
\left(\mathrm{gCO}_{2} \mathrm{e} / \mathrm{kWh}\right)\end{array}$} & \multirow{2}{*}{$\begin{array}{c}\text { Additional Features } \\
\text { EGC (MW); CF (\%); PL (years); GL }\end{array}$} \\
\hline & $\begin{array}{l}\text { Facility } \\
\text { Type }\end{array}$ & $\begin{array}{c}\text { Electric Power } \\
\text { Generation Capacity }\end{array}$ & $\begin{array}{c}\text { Unique } \\
\text { Category Code }\end{array}$ & & \\
\hline \multirow{38}{*}{ Varun et al. [15] } & $\mathrm{D}$ & $\mathrm{S}$ & D-S & 24.57 & EGC $=5(2 \times 2.5) ; \mathrm{PL}=30 ; \mathrm{GL}=$ Uttarakhand, India \\
\hline & $\mathrm{D}$ & $\mathrm{s}$ & D-S & 21.43 & $\mathrm{EGC}=8(2 \times 4) ; \mathrm{PL}=30 ; \mathrm{GL}=$ Arunachal Pradesh, India \\
\hline & $\mathrm{D}$ & $\mathrm{S}$ & D-S & 29.19 & $\mathrm{EGC}=2(2 \times 1) ; \mathrm{PL}=30 ; \mathrm{GL}=$ Sikkim, India \\
\hline & $\mathrm{D}$ & $\mathrm{S}$ & D-S & 27.94 & $\mathrm{EGC}=3(3 \times 1) ; \mathrm{PL}=30 ; \mathrm{GL}=$ Uttarakhand, India \\
\hline & $\mathrm{D}$ & $\mathrm{s}$ & D-S & 33.66 & $\mathrm{EGC}=1(2 \times 0.5) ; \mathrm{PL}=30 ; \mathrm{GL}=$ Sikkim, India \\
\hline & $\mathrm{D}$ & $\mathrm{S}$ & D-S & 23.68 & $\mathrm{EGC}=5(2 \times 2.5) ; \mathrm{PL}=30 ; \mathrm{GL}=$ Sikkim, India \\
\hline & $\mathrm{D}$ & $\mathrm{S}$ & D-S & 20.5 & $\mathrm{EGC}=9(3 \times 3) ; \mathrm{PL}=30 ; \mathrm{GL}=$ Sikkim, India \\
\hline & $\mathrm{D}$ & $\mathrm{S}$ & D-S & 27.89 & $\mathrm{EGC}=2(2 \times 1) ; \mathrm{PL}=30 ; \mathrm{GL}=$ Arunachal Pradesh, India \\
\hline & $\mathrm{D}$ & $\mathrm{S}$ & D-S & 21.12 & $\mathrm{EGC}=6(3 \times 2) ; \mathrm{PL}=30 ; \mathrm{GL}=$ Arunachal Pradesh, India \\
\hline & $\mathrm{D}$ & $\mathrm{S}$ & D-S & 31.46 & EGC $=0.9(3 \times 0.3) ; \mathrm{PL}=30 ; \mathrm{GL}=$ Jammu \& Kashmir, India \\
\hline & $\mathrm{D}$ & $\mathrm{s}$ & D-S & 18.87 & EGC $=4(2 \times 2) ; \mathrm{PL}=30 ; \mathrm{GL}=$ Himachal Pradesh, India \\
\hline & $\mathrm{D}$ & $\mathrm{S}$ & D-S & 23.17 & $\mathrm{EGC}=2(2 \times 1) ; \mathrm{PL}=30 ; \mathrm{GL}=$ Himachal Pradesh, India \\
\hline & $\mathrm{D}$ & $\mathrm{s}$ & D-S & 48.23 & EGC $=0.2(2 \times 0.1) ; \mathrm{PL}=30 ; \mathrm{GL}=$ Himachal Pradesh, India \\
\hline & $\mathrm{D}$ & $\mathrm{S}$ & D-S & 20.18 & $\mathrm{EGC}=4.5(2 \times 2.25) ; \mathrm{PL}=30 ; \mathrm{GL}=$ Himachal Pradesh, India \\
\hline & $\mathrm{D}$ & $\mathrm{S}$ & D-S & 37.52 & $\mathrm{EGC}=1(2 \times 0.5) ; \mathrm{PL}=30 ; \mathrm{GL}=$ Uttarakhand, India \\
\hline & $\mathrm{D}$ & $\mathrm{s}$ & D-S & 29.12 & $\mathrm{EGC}=2(2 \times 1) ; \mathrm{PL}=30 ; \mathrm{GL}=$ Sikkim, India \\
\hline & $\mathrm{D}$ & $\mathrm{S}$ & D-S & 25.05 & $\mathrm{EGC}=4.5(2 \times 2.25) ; \mathrm{PL}=30 ; \mathrm{GL}=$ Himachal Pradesh, India \\
\hline & $\mathrm{D}$ & $\mathrm{S}$ & D-S & 31.2 & EGC $=1(2 \times 0.5) ; \mathrm{PL}=30 ; \mathrm{GL}=$ Himachal Pradesh, India \\
\hline & $\mathrm{D}$ & $\mathrm{S}$ & D-S & 32.57 & EGC $=0.9(2 \times 0.45) ; \mathrm{PL}=30 ; \mathrm{GL}=$ Himachal Pradesh, India \\
\hline & $\mathrm{D}$ & $\mathrm{S}$ & D-S & 34.03 & $\mathrm{EGC}=0.8 ; \mathrm{PL}=30 ; \mathrm{GL}=$ Himachal Pradesh, India \\
\hline & $\mathrm{D}$ & $\mathrm{S}$ & D-S & 29 & $\mathrm{EGC}=3(2 \times 1.5) ; \mathrm{PL}=30 ; \mathrm{GL}=$ Himachal Pradesh, India \\
\hline & $\mathrm{D}$ & $\mathrm{S}$ & D-S & 24.22 & $\mathrm{EGC}=3(2 \times 1.5) ; \mathrm{PL}=30 ; \mathrm{GL}=$ Himachal Pradesh, India \\
\hline & $\mathrm{D}$ & $\mathrm{S}$ & D-S & 22.99 & $\mathrm{EGC}=3(2 \times 1.5) ; \mathrm{PL}=30 ; \mathrm{GL}=$ Himachal Pradesh, India \\
\hline & $\mathrm{D}$ & $\mathrm{S}$ & D-S & 23.99 & $\mathrm{EGC}=5(2 \times 2.5) ; \mathrm{PL}=30 ; \mathrm{GL}=$ Himachal Pradesh, India \\
\hline & $\mathrm{D}$ & $\mathrm{S}$ & D-S & 21.12 & $\mathrm{EGC}=3(2 \times 1.5) ; \mathrm{PL}=30 ; \mathrm{GL}=$ Himachal Pradesh, India \\
\hline & $\mathrm{D}$ & $\mathrm{S}$ & D-S & 26.7 & $\mathrm{EGC}=1.8(2 \times 0.9) ; \mathrm{PL}=30 ; \mathrm{GL}=$ Himachal Pradesh, India \\
\hline & $\mathrm{D}$ & $\mathrm{S}$ & D-S & 27.12 & EGC $=1(2 \times 0.5) ; \mathrm{PL}=30 ; \mathrm{GL}=$ Himachal Pradesh, India \\
\hline & $\mathrm{D}$ & $\mathrm{S}$ & D-S & 30.58 & $\mathrm{EGC}=1(2 \times 0.5) ; \mathrm{PL}=30 ; \mathrm{GL}=$ Himachal Pradesh, India \\
\hline & $\mathrm{D}$ & $\mathrm{S}$ & D-S & 28.71 & EGC $=1(2 \times 0.5) ; \mathrm{PL}=30 ; \mathrm{GL}=$ Himachal Pradesh, India \\
\hline & $\mathrm{D}$ & $\mathrm{S}$ & D-S & 22.66 & $\mathrm{EGC}=3(2 \times 1.5) ; \mathrm{PL}=30 ; \mathrm{GL}=$ Himachal Pradesh, India \\
\hline & $\mathrm{D}$ & $\mathrm{S}$ & D-S & 14 & $\mathrm{EGC}=21 ; \mathrm{PL}=30 ; \mathrm{GL}=$ Kerala India \\
\hline & $\mathrm{D}$ & $\mathrm{S}$ & D-S & 25.11 & $\mathrm{EGC}=2(2 \times 1) ; \mathrm{PL}=30 ; \mathrm{GL}=$ Uttar Pradesh, India \\
\hline & $\mathrm{D}$ & $\mathrm{S}$ & D-S & 21.03 & $\mathrm{EGC}=3(3 \times 1) ; \mathrm{PL}=30 ; \mathrm{GL}=$ Uttar Pradesh, India \\
\hline & $\mathrm{D}$ & $\mathrm{S}$ & D-S & 20.58 & $\mathrm{EGC}=3(3 \times 1) ; \mathrm{PL}=30 ; \mathrm{GL}=$ Uttarakhand, India \\
\hline & $\mathrm{D}$ & $\mathrm{S}$ & D-S & 22.85 & $\mathrm{EGC}=3(3 \times 1) ; \mathrm{PL}=30 ; \mathrm{GL}=$ Uttarakhand, India \\
\hline & $\mathrm{D}$ & $\mathrm{s}$ & D-S & 28.12 & $\mathrm{EGC}=1(2 \times 0.5) ; \mathrm{PL}=30 ; \mathrm{GL}=$ Uttarakhand, India \\
\hline & $\mathrm{D}$ & $\mathrm{S}$ & D-S & 22.01 & $\mathrm{EGC}=3(3 \times 1) ; \mathrm{PL}=30 ; \mathrm{GL}=$ Uttarakhand, India \\
\hline & $\mathrm{D}$ & $\mathrm{S}$ & D-S & 27.16 & $\mathrm{EGC}=3(2 \times 1.5) ; \mathrm{PL}=30 ; \mathrm{GL}=$ Uttarakhand, India \\
\hline
\end{tabular}


Table 1. Cont.

\begin{tabular}{|c|c|c|c|c|c|}
\hline \multirow[b]{2}{*}{ Source } & \multicolumn{3}{|c|}{ Categorization } & \multirow{2}{*}{$\begin{array}{l}\text { GHG Emissions } \\
\left(\mathrm{gCO}_{2} \mathrm{e} / \mathrm{kWh}\right)\end{array}$} & \multirow{2}{*}{$\begin{array}{c}\text { Additional Features } \\
\text { EGC (MW); CF (\%); PL (years); GL }\end{array}$} \\
\hline & $\begin{array}{l}\text { Facility } \\
\text { Type }\end{array}$ & $\begin{array}{c}\text { Electric Power } \\
\text { Generation Capacity }\end{array}$ & $\begin{array}{c}\text { Unique } \\
\text { Category Code }\end{array}$ & & \\
\hline \multirow{22}{*}{ Varun et al. [15] } & $\mathrm{D}$ & $\mathrm{S}$ & D-S & 26.63 & $\mathrm{EGC}=6(2 \times 3) ; \mathrm{PL}=30 ; \mathrm{GL}=\mathrm{Uttarakhand}$, India \\
\hline & $\mathrm{D}$ & $\mathrm{s}$ & D-S & 49.18 & $\mathrm{EGC}=0.3(3 \times 0.1) ; \mathrm{PL}=30 ; \mathrm{GL}=\mathrm{Jammu} \& \mathrm{Kashmir}$, India \\
\hline & $\mathrm{D}$ & $\mathrm{S}$ & D-S & 43.36 & EGC $=0.6(3 \times 0.2) ; \mathrm{PL}=30 ; \mathrm{GL}=$ Himachal Pradesh, India \\
\hline & $\mathrm{D}$ & $\mathrm{s}$ & D-S & 29.18 & $\mathrm{EGC}=6(2 \times 3) ; \mathrm{PL}=30 ; \mathrm{GL}=$ Arunachal Pradesh, India \\
\hline & $\mathrm{D}$ & $\mathrm{S}$ & D-S & 43.06 & EGC $=0.75(3 \times 0.25) ; \mathrm{PL}=30 ; \mathrm{GL}=$ Arunachal Pradesh, India \\
\hline & $\mathrm{D}$ & $\mathrm{S}$ & D-S & 41.91 & EGC $=0.75(3 \times 0.25) ; \mathrm{PL}=30 ; \mathrm{GL}=$ Arunachal Pradesh, India \\
\hline & $\mathrm{D}$ & $\mu$ & $D-\mu$ & 74.87 & $\mathrm{EGC}=0.05(2 \times 0.025) ; \mathrm{PL}=30 ; \mathrm{GL}=$ Uttarakhand, India \\
\hline & $\mathrm{D}$ & $\mathrm{s}$ & D-S & 55.87 & EGC $=0.1(2 \times 0.05) ; \mathrm{PL}=30 ; \mathrm{GL}=$ Uttarakhand, India \\
\hline & $\mathrm{D}$ & $\mathrm{s}$ & D-S & 25.85 & $\mathrm{EGC}=6(2 \times 3) ; \mathrm{PL}=30 ; \mathrm{GL}=$ Arunachal Pradesh, India \\
\hline & $\mathrm{D}$ & $\mu$ & $D-\mu$ & 59.65 & EGC $=0.05(2 \times 0.025) ; \mathrm{PL}=30 ; \mathrm{GL}=$ Uttarakhand, India \\
\hline & $\mathrm{D}$ & $\mathrm{S}$ & $\mathrm{D}-\mathrm{S}$ & 62.86 & $\mathrm{EGC}=0.1(2 \times 0.05) ; \mathrm{PL}=30 ; \mathrm{GL}=$ Uttarakhand, India \\
\hline & $\mathrm{D}$ & $\mathrm{s}$ & D-S & 49.24 & $\mathrm{EGC}=0.2(2 \times 0.1) ; \mathrm{PL}=30 ; \mathrm{GL}=\mathrm{Uttarakhand}$, India \\
\hline & $\mathrm{D}$ & $\mathrm{s}$ & D-S & 21.98 & $\mathrm{EGC}=4 ; \mathrm{PL}=30 ; \mathrm{GL}=$ Maharashtra, India \\
\hline & $\mathrm{D}$ & $\mathrm{S}$ & D-S & 15.96 & $\mathrm{EGC}=25(2 \times 12.5) ; \mathrm{PL}=30 ; \mathrm{GL}=$ Orissa, India \\
\hline & $\mathrm{D}$ & $\mathrm{s}$ & D-S & 18.26 & $\mathrm{EGC}=12(3 \times 4) ; \mathrm{PL}=30 ; \mathrm{GL}=$ Orissa, India \\
\hline & $\mathrm{D}$ & $\mathrm{S}$ & D-S & 27.24 & $\mathrm{EGC}=3(2 \times 1.5) ; \mathrm{PL}=30 ; \mathrm{GL}=$ Uttarakhand, India \\
\hline & $\mathrm{D}$ & $\mathrm{S}$ & D-S & 26.95 & $\mathrm{EGC}=3(2 \times 1.5) ; \mathrm{PL}=30 ; \mathrm{GL}=$ Uttarakhand, India \\
\hline & $\mathrm{D}$ & $\mathrm{s}$ & D-S & 22.76 & $\mathrm{EGC}=3(2 \times 1.5) ; \mathrm{PL}=30 ; \mathrm{GL}=$ Uttarakhand, India \\
\hline & $\mathrm{D}$ & $\mathrm{S}$ & D-S & 32.49 & $\mathrm{EGC}=3(2 \times 1.5) ; \mathrm{PL}=30 ; \mathrm{GL}=$ Arunachal Pradesh, India \\
\hline & $\mathrm{D}$ & $\mathrm{s}$ & D-S & 74.79 & $\mathrm{EGC}=2(2 \times 1) ; \mathrm{PL}=30 ; \mathrm{GL}=$ Arunachal Pradesh, India \\
\hline & $\mathrm{D}$ & $\mathrm{s}$ & D-S & 31.74 & $\mathrm{EGC}=3(3 \times 1) ; \mathrm{PL}=30 ; \mathrm{GL}=\mathrm{Uttar}$ Pradesh, India \\
\hline & $\mathrm{D}$ & $\mathrm{S}$ & D-S & 35.49 & EGC $=3(3 \times 1) ; \mathrm{PL}=30 ; \mathrm{GL}=$ Uttarakhand, India \\
\hline Arnøy and Modahl [16] & $\mathrm{D}$ & $\mathrm{L}$ & D-L & 2.19 & EGC $=52.5 ; \mathrm{PL}=100 ; \mathrm{GL}=$ Embretsfoss, Norway \\
\hline Arnøy and Modahl [17] & I & $\mathrm{L}$ & $\mathrm{I}-\mathrm{L}$ & 0.61 & EGC $=130 ; \mathrm{PL}=100 ; \mathrm{GL}=$ Trollheim, Norway \\
\hline \multirow{2}{*}{ Donnelly et al. [18] } & $\mathrm{I}$ & $\mathrm{L}$ & I-L & 15 & $\mathrm{EGC}=1000 ; \mathrm{CF}=57.5 ; \mathrm{PL}=100$ \\
\hline & $\mathrm{D}$ & $\mathrm{L}$ & D-L & 5 & $\mathrm{EGC}=100 ; \mathrm{CF}=57.5 ; \mathrm{PL}=100$ \\
\hline Environmental Product Declaration Report [19] & $\mathrm{D}$ & $\mathrm{S}$ & D-S & 5.23 & EGC $=2.1 ; P L=100 ; G L=$ Zürich, Switzerland \\
\hline \multirow{3}{*}{ Gallagher et al. [20] } & $\mathrm{D}$ & S & D-S & 5.46 & EGC $=0.65 ; \mathrm{PL}=50 ; \mathrm{GL}=$ North Wales, $\mathrm{UK}$ \\
\hline & $\mathrm{D}$ & $\mathrm{S}$ & D-S & 7.39 & $\mathrm{EGC}=0.1 ; \mathrm{PL}=50 ; \mathrm{GL}=$ North Wales, $\mathrm{UK}$ \\
\hline & $\mathrm{D}$ & $\mu$ & $D-\mu$ & 8.93 & $\mathrm{EGC}=0.05 ; \mathrm{PL}=50 ; \mathrm{GL}=$ North England, $\mathrm{UK}$ \\
\hline \multirow{3}{*}{ Gallagher et al. [21] } & MHPW & $\mu$ & MHPW- $\mu$ & 2.14 & $\mathrm{EGC}=0.015 ; \mathrm{PL}=30 ; \mathrm{GL}=\mathrm{UK}$ \\
\hline & MHPW & $\mu$ & MHPW- $\mu$ & 4.36 & $\mathrm{EGC}=0.09 ; \mathrm{PL}=30 ; \mathrm{GL}=$ Ireland \\
\hline & MHPW & S & MHPW-S & 2.78 & $\mathrm{EGC}=0.14 ; \mathrm{PL}=30 ; \mathrm{GL}=\mathrm{UK}$ \\
\hline Hanafi and Riman [22] & $\mathrm{D}$ & S & D-S & 1.2 & $\mathrm{EGC}=9 ; \mathrm{PL}=50 ; \mathrm{GL}=$ Simalungun, Indonesia \\
\hline
\end{tabular}




\subsection{Statistical Evaluation of Hydro LCA Studies}

Figure 1 provides a graphical representation of the (a) error bars (mean $\pm 95 \%$ confidence interval (CI) statistics) and (b) box plots (quartiles + outlier statistics) for GHG emissions from the different hydroelectricity generation systems reviewed in this study. Table 2 provides a statistical summary of the life cycle GHG emissions with details on the sample size $(\mathrm{N})$, mean $(\mathrm{X}) \pm$ standard deviation (SD), minimum (Min.), maximum (Max.), standard error of the mean (SE), quartile 1 (Q1), quartile 2 or median $(\mathrm{Q} 2)$, and quartile $3(\mathrm{Q} 3)$ for the different hydroelectricity generation systems reviewed in this study.

From Figure 1a and Table 2, one may note that the mean life cycle GHG emissions obtained from the use of I-S, I-L, D- $\mu$, D-S, D-L, PS-L, MHPW- $\mu$, and MHPW-S hydroelectricity generation systems are $21.05 \mathrm{gCO}_{2} \mathrm{e} / \mathrm{kWh}, 40.63 \mathrm{gCO}_{2} \mathrm{e} / \mathrm{kWh}, 47.82 \mathrm{gCO}_{2} \mathrm{e} / \mathrm{kWh}, 27.18 \mathrm{gCO}_{2} \mathrm{e} / \mathrm{kWh}, 3.45 \mathrm{gCO}_{2} \mathrm{e} / \mathrm{kWh}$, $256.63 \mathrm{gCO}_{2} \mathrm{e} / \mathrm{kWh}, 19.73 \mathrm{gCO}_{2} \mathrm{e} / \mathrm{kWh}$, and $2.78 \mathrm{gCO}_{2} \mathrm{e} / \mathrm{kWh}$, respectively. In the case of impoundment hydroelectricity generation systems, the mean life cycle GHGs emitted from large hydroelectricity generation systems (I-L) was more than the mean life cycle GHGs emitted from small hydroelectricity generation systems (I-S). The higher mean life cycle GHG emissions from large electricity generation capacity impoundment systems may be attributed to the activities of construction (mining, steel, concrete) and the flooding of vegetated land [15,23]. Additionally, one may also note that the use of an alternative back-up power generation system (e.g., thermal power generation system) to account for the seasonal variations in hydroelectricity generation supply can result in a significant increase of the life cycle GHG emissions from the I-L hydroelectricity generation systems $[4,23]$. Two studies $[15,28]$ noted the life cycle GHG emissions from impoundment hydroelectricity generation systems to be directly proportional to the gross static head (distance between the intake and the turbine).

In the case of diversion hydroelectricity generation systems, the mean life cycle GHG emissions from micro hydroelectricity generation systems (D- $\mu$ ) was more than the mean life cycle GHG emissions from small hydroelectricity generation systems (D-S) and large hydroelectricity generation systems (D-L). The mean life cycle GHG emissions decreased with an increase in the electricity generation capacity for diversion hydroelectricity generation systems. From Figure 1a and Table 2, one may note that the mean life cycle GHG emissions were minimum for the D-L hydroelectricity generation systems (considering the hydroelectricity generation system categories with a representation of at least two cases). The diversion hydroelectricity generation systems emitted less GHGs in comparison to the impoundment hydroelectricity generation systems across the large electricity generation capacity category (refer to Figure 1a and Table 2). Similar observations were made by another study that noted the run-off hydroelectricity generation systems to emit lower GHG emissions than the impoundment hydroelectricity generation systems [23].

The mean life cycle GHG emissions were found to be the highest for PS-L hydroelectricity generation systems. The higher mean life cycle GHG emissions for PS-L hydroelectricity generation systems may be attributed to the higher cumulative energy demand (approximately three times the cumulative energy demand required for diversion and impoundment hydroelectricity generation systems) for consumption by the pumps [13].

The mean life cycle GHG emissions from miscellaneous hydropower works was less than the mean life cycle GHG emissions from diversion hydroelectricity generation systems across the micro electricity generation capacity category (refer to Figure 1a and Table 2). The lower GHG emissions from miscellaneous hydropower works may be attributed to the requirement of less construction activities than in the case of diversion hydroelectricity generation systems. The use of existing water infrastructures to generate electricity at micro levels for local consumption was noted to be an environmental-friendly option that needs more investigation in the future. More LCA studies utilizing MHPW-S hydroelectricity generation systems are to be considered before one generalizes the influence of MHPW-S on the mean life cycle GHG emissions. 


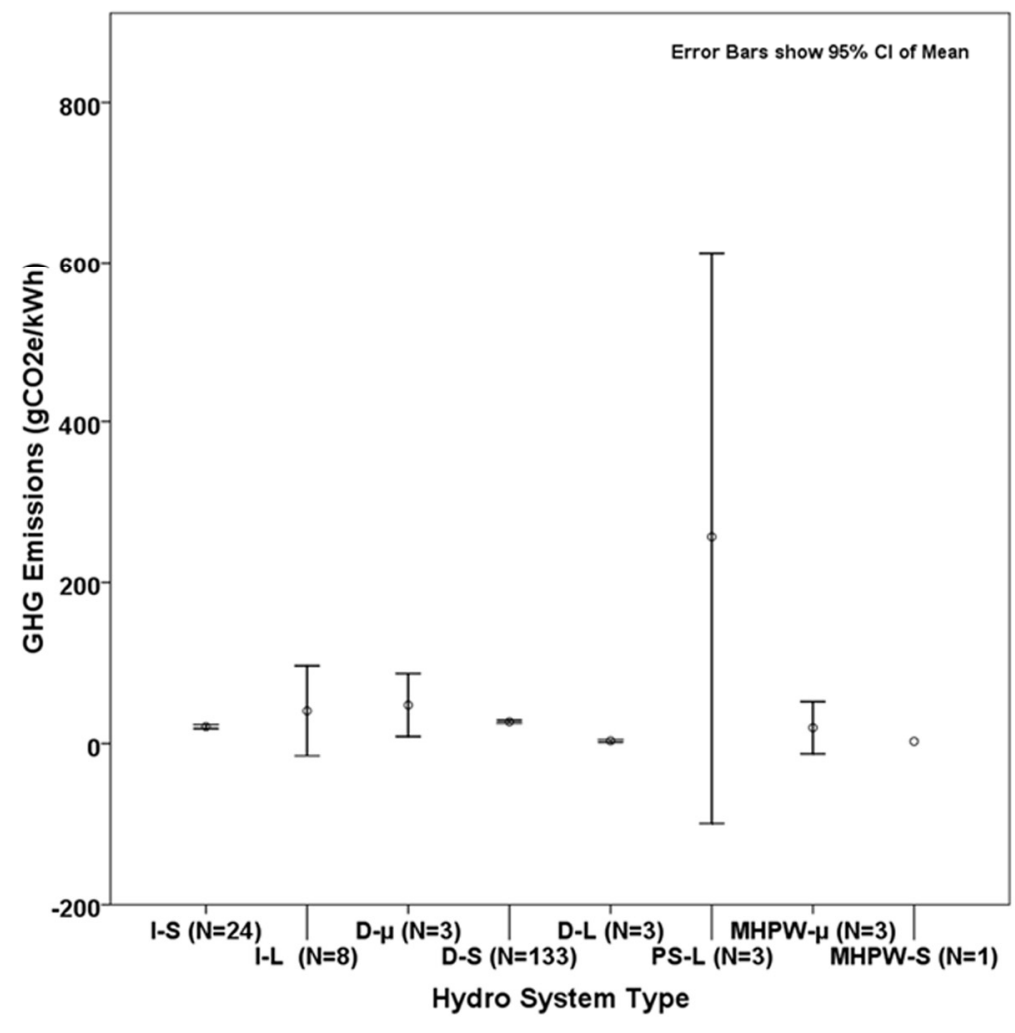

(a)

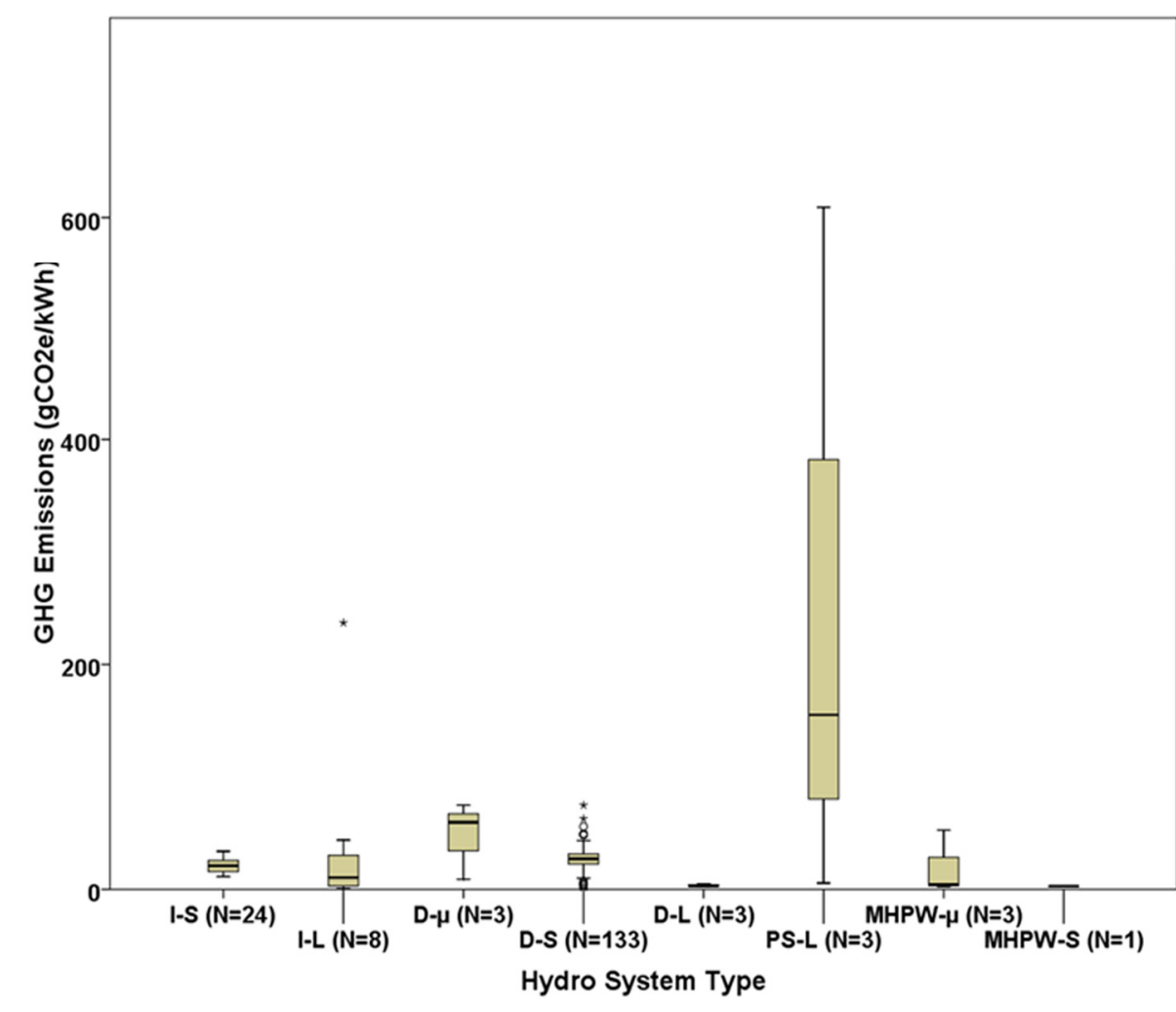

(b)

Figure 1. GHG emissions from hydroelectricity generation systems: (a) mean $\pm 95 \%$ CI error bars and (b) quartile box plots. 
Table 2. GHG emission $\left(\mathrm{gCO}_{2} \mathrm{e} / \mathrm{kWh}\right)$ statistics from hydroelectricity generation systems.

\begin{tabular}{ccccccccc}
\hline Hydro System Type & $\mathbf{N}$ & $\mathbf{X} \pm \mathbf{S D}$ & $\mathbf{M i n}$. & $\mathbf{M a x}$. & $\mathbf{S E}$ & $\mathbf{Q 1}$ & $\mathbf{Q 2}$ & Q3 \\
\hline I-S & 24 & $21.05 \pm 6.25$ & 11.34 & 33.86 & 1.28 & 15.25 & 21.01 & 25.6 \\
I-L & 8 & $40.63 \pm 80.57$ & 0.61 & 237 & 28.49 & 1.56 & 10.43 & 16.64 \\
D- $\mu$ & 3 & $47.82 \pm 34.53$ & 8.93 & 74.87 & 19.93 & 8.93 & 59.65 & 74.87 \\
D-S & 133 & $27.18 \pm 10.38$ & 1.2 & 74.79 & 0.9 & 22.66 & 27.1 & 31.56 \\
D-L & 3 & $3.45 \pm 1.43$ & 2.19 & 5 & 0.82 & 2.19 & 3.16 & 5 \\
PS-L & 3 & $256.63 \pm 314.35$ & 5.6 & 609.2 & 181.49 & 5.6 & 155.1 & 155.1 \\
MHPW- $\mu$ & 3 & $19.73 \pm 28.57$ & 2.14 & 52.7 & 16.5 & 2.14 & 4.36 & 52.7 \\
MHPW-S & 1 & $2.78 \pm 0$ & 2.78 & 2.78 & 0 & 2.78 & 2.78 & 2.78 \\
\hline
\end{tabular}

From Figure 1b, one may note that the degree of variation in GHG emissions was minimal for D-L hydroelectricity generation systems, followed by D-S, I-S, I-L, MHPW- $\mu, \mathrm{D}-\mu$, and PS-L hydroelectricity generation systems. The degree of variation being less indicates the level of maturity and consistency in the use advanced technology in the various stages of life cycle analysis, irrespective of the geographical locations and many other influential factors. Note that the standard deviation values for I-L and PS-L hydroelectricity generation systems are more than the corresponding mean values (refer to Table 2), thereby indicating that there is a large variation in the use of technology across different geographic locations. In the context of both the mean life cycle GHG emissions and the degree of variation in GHG emissions from D-L hydroelectricity generation systems being minimal, this study identifies the diversion hydroelectricity generation systems with large electricity generation capacity to be the most environmental-friendly option amongst the different categories of hydroelectricity generation systems considered in this study.

\section{Conclusions}

This paper evaluated the life cycle GHG emissions from hydroelectricity generation systems by developing a new categorization index that integrated the names of the two classification systems based on the facility type (I, D, PS, MHPW) and the electric power generation capacity ( $\mu, S, L)$. A total of 19 hydroelectricity generation system LCA studies that summarized 178 representative cases were identified in the literature and reviewed in this study. Each of the reviewed hydroelectricity generation system LCA studies was first assigned a unique category code in accordance with the developed categorization index. The categorization of the reviewed 19 hydroelectricity generation system LCA studies yielded a total of eight distinct categories, namely, I-S, I-L, D- $\mu$, D-S, D-L, PS-L, MHPW- $\mu$, and MHPW-S.

The mean life cycle GHG emissions from I-S, I-L, D- $\mu$, D-S, D-L, PS-L, MHPW- $\mu$, and MHPW-S hydroelectricity generation systems are $21.05 \mathrm{gCO}_{2} \mathrm{e} / \mathrm{kWh}, 40.63 \mathrm{gCO}_{2} \mathrm{e} / \mathrm{kWh}, 47.82 \mathrm{gCO} 2 \mathrm{e} / \mathrm{kWh}$ $27.18 \mathrm{gCO}_{2} \mathrm{e} / \mathrm{kWh}, 3.45 \mathrm{gCO}_{2} \mathrm{e} / \mathrm{kWh}, 256.63 \mathrm{gCO}_{2} \mathrm{e} / \mathrm{kWh}, 19.73 \mathrm{gCO}_{2} \mathrm{e} / \mathrm{kWh}$, and $2.78 \mathrm{gCO}_{2} \mathrm{e} / \mathrm{kWh}$ respectively. The mean life cycle GHG emissions from I-L hydroelectricity generation systems were noted to higher than the mean life cycle GHG emissions from I-S electric generation systems (the relatively higher GHG emissions for I-L hydroelectricity generation systems being attributed to the dam construction activities, the flooding of vegetated land, and the use of back-up power). The mean life cycle GHGs emitted from D-L hydroelectricity generation systems was more than the mean life cycle GHGs emitted from D-S and D- $\mu$ hydroelectricity generation systems. For the case of large hydroelectricity generation systems, the mean life cycle GHG emissions from diversion hydroelectricity generation systems was less than the mean life cycle GHG emissions from impoundment hydroelectricity generation systems. D-L hydroelectricity generation systems provided the most environmental-friendly option with the lowest mean life GHG emissions and minimal variations between the reviewed D-L LCA case representations (considering only the hydroelectricity generation system categories with a representation of at least two cases). PS-L hydroelectricity generation systems had the highest mean life cycle GHG emissions due to the 
higher energy consumption by pumps. For micro electricity generation capacity, the miscellaneous hydropower works emitted less GHGs than the diversion hydroelectricity generation systems due to limited emissions from construction activities. There was only one LCA case representation noted in the literature for MHPW-S systems. Future research needs to focus on the development of more hydro LCA studies (that includes comprehensive details on the site-specific characteristics of the hydroelectricity system) with emphasis on MHPW-S systems.

Acknowledgments: This work was supported by the US National Science Foundation through the CREST Center for Energy and Environmental Sustainability (CEES) at Prairie View A\&M University, award number 1036593.

Author Contributions: Akhil Kadiyala conceived the approach to scrutinize hydroelectricity life cycle GHG emissions in-detail and developed the manuscript under the guidance of Professors Raghava Kommalapati and Ziaul Huque.

Conflicts of Interest: The authors declare no conflict of interest.

\section{References}

1. Wagner, H.J.; Mathur, J. Introduction to Hydro Energy Systems: Basics, Technology and Operation; Springer: New York, NY, USA, 2011; pp. 49-52.

2. International Energy Statistics. Available online: http://www.eia.gov/cfapps/ipdbproject/iedindex3.cfm? tid $=44 \&$ pid $=44 \&$ aid $=2 \& c i d=w w, r 1, \& s y i d=2008 \&$ eyid $=2012 \& u n i t=Q B T U$ (accessed on 1 June 2016).

3. United States Energy Information Administration. International Energy Outlook 2013. Available online: http:/ /www.eia.gov/forecasts/ieo/pdf/0484(2013).pdf (accessed on 1 June 2016).

4. Gagnon, L.; van de Vate, J.F. Greenhouse gas emissions from hydropower: The state of research in 1996. Energy Policy 1997, 25, 7-13. [CrossRef]

5. Denholm, P.; Kulcinski, G.L. Life cycle energy requirements and greenhouse gas emissions from large scale energy storage systems. Energy Convers. Manag. 2004, 45, 2153-2172. [CrossRef]

6. Hondo, H. Life cycle GHG emission analysis of power generation systems: Japanese case. Energy 2005, 30, 2042-2056. [CrossRef]

7. Pehnt, M. Dynamic life cycle assessment (LCA) of renewable energy technologies. Renew. Energy 2006, 31, 55-71. [CrossRef]

8. Rule, B.M.; Worth, Z.J.; Boyle, C.A. Comparison of life cycle carbon dioxide emissions and embodied energy in four renewable electricity generation technologies in New Zealand. Environ. Sci. Technol. 2009, 43, 6406-6413. [CrossRef] [PubMed]

9. Environmental Product Declaration: Wildegg-Brugg Run-of-River Power Plant. Available online: http:// gryphon.environdec.com/data/files/6/9037/epd205_Axpo_Wildegg_Brugg.pdf (accessed on 1 June 2016).

10. Ribeiro, F.M.; da Silva, G.A. Life-cycle inventory for hydroelectric generation: A Brazilian case study. J. Clean. Prod. 2010, 18, 44-54. [CrossRef]

11. Pascale, A.; Urmee, T.; Moore, A. Life cycle assessment of a community hydroelectric power system in rural Thailand. Renew. Energy 2011, 36, 2799-2808. [CrossRef]

12. Suwanit, W.; Gheewala, S.H. Life cycle assessment of mini-hydropower plants in Thailand. Int. J. Life Cycle Assess. 2011, 16, 849-858. [CrossRef]

13. Flury, K.; Frischknecht, R. Life Cycle Inventories of Hydroelectric Power Generation. Available online: http://www.esu-services.ch/fileadmin/download/publicLCI/flury-2012-hydroelectric-powergeneration.pdf (accessed on 1 June 2016).

14. National Energy Technology Laboratory. Role of Alternative Energy Sources: Hydropower Technology Assessment. Available online: https://www.netl.doe.gov/energy-analyses/temp/ FY12_RoleofAlternativeEnergySourcesHydropowerTechnologyAssessmentBrief_080112.pdf (accessed on 13 May 2016).

15. Varun; Prakash, R.; Bhat, I.K. Life cycle greenhouse gas emissions estimation for small hydropower schemes in India. Energy 2012, 44, 498-508. [CrossRef] 
16. Arnøy, S.; Modahl, I.S. Life Cycle Data for Hydroelectric Generation at Embretsfoss 4 (E4) Power Station: Background Data for Life Cycle Assessment (LCA) and Environmental Product Declaration (EPD) 2013. Available online: http:/ / ostfoldforskning.no/uploads/dokumenter/publikasjoner/703.pdf (accessed on 9 May 2016).

17. Arnøy, S.; Modahl, I.S. Life Cycle Data for Hydroelectric Generation at Trollheim Power Station: Background Data for Updating Environmental Product Declaration (EPD) 2013. Available online: http:/ /ostfoldforskning. no/uploads/dokumenter/publikasjoner/722.pdf (accessed on 9 May 2016).

18. Donnelly, C.R.; Carias, A.; Morgenroth, M.; Ali, M.; Bridgeman, A.; Wood, N. An assessment of the life cycle costs and GHG emissions for alternative generation technologies. In Proceedings of the 2010 International World Energy Council Conference, Montreal, Quebec, Canada, 12-16 September 2010.

19. Environmental Product Declaration: Au-Schönenberg Small-Scale Hydro Power Plant. Available online: https://www.axpo.com/content/dam/axpo/switzerland/umwelt/dokumente/umweltdeklaration_au_ schoenenberg_2014_en.pdf (accessed on 1 June 2016).

20. Gallagher, J.; Styles, D.; McNabola, A.; Williams, A.P. Current and future environmental balance of small-scale run-of-river hydropower. Environ. Sci. Technol. 2015, 49, 6344-6351. [CrossRef] [PubMed]

21. Gallagher, J.; Styles, D.; McNabola, A.; Williams, A.P. Life cycle environmental balance and greenhouse gas mitigation potential of micro-hydropower energy recovery in the water industry. J. Clean. Prod. 2015, 99, 152-159. [CrossRef]

22. Hanafi, J.; Riman, A. Life cycle assessment of a mini hydro power plant in Indonesia: A case study in Karai river. Procedia CIRP 2015, 29, 444-449. [CrossRef]

23. Amponsah, N.W.; Troldborg, M.; Kington, B.; Aalders, I.; Hough, R.L. Greenhouse gas emissions from renewable energy sources: A review of lifecycle considerations. Renew. Sustain. Energy Rev. 2014, 39, 461-475. [CrossRef]

24. Asdrubali, F.; Baldinelli, G.; D’Alessandro, F.; Scrucca, F. Life cycle assessment of electricity production from renewable energies: Review and results harmonization. Renew. Sustain. Energy Rev. 2015, 42, 1113-1122. [CrossRef]

25. Raadal, H.L.; Gagnon, L.; Modahl, I.S.; Hanssen, O.J. Life cycle greenhouse gas (GHG) emissions from the generation of wind and hydro power. Renew. Sustain. Energy Rev. 2011, 15, 3417-3422. [CrossRef]

26. Turconi, R.; Boldrin, A.; Astrup, T. Life cycle assessment (LCA) of electricity generation technologies: Overview, comparability and limitations. Renew. Sustain. Energy Rev. 2013, 28, 555-565. [CrossRef]

27. Types of Hydropower Plants. Available online: http://energy.gov/eere/water/types-hydropower-plants (accessed on 1 June 2016).

28. Gleick, P.H. Environmental consequences of hydroelectric development: The role of facility size and type. Energy 1992, 17, 735-747. [CrossRef] 